\title{
La integración de lengua y contenidos afines a diferentes carreras universitarias
}

\author{
Integrating language and content related to different university \\ degree programs
}

\author{
Pablo Marcelo Oliva Parera \\ Monterey Institute of International Studies \\ (Monterey, CA, USA) \\ Maria Pilar Nuñez Delgado \\ University of Granada \\ (Granada, Spain)
}

\begin{abstract}
This article shows the description of an experience involving a group of graduate-level university students taking a CLIL (content and language integrated learning) course in Spanish as a foreign language. An important contribution from this study is to show that it is possible to achieve the integration of content and language by incorporating the content of the students' interests related to the concentrations of their master's degrees. This group of students is taking a Spanish class at the low and mid-intermediate level (ACTFL, 2012). Their fields of their Master's programs varied from international policy, to environmental studies, to business. The students had the opportunity to work with content related to their programs over the course of a semester. The instructor used an adaptation of the European Language Portfolio in order to measure part of their performance in class.
\end{abstract}

Key Words: autonomy; curriculum development; portfolios; content and language integrated learning; motivation.

\section{Resumen}

En este artículo se presenta la descripción de una experiencia por parte de un grupo de estudiantes de nivel universitario al currículo de una clase AICLE (Aprendizaje Integrado de Contenidos y de Lengua Extranjera) en español. Un aporte en esta dirección lo constituye el tener en cuenta los intereses de los estudiantes, o como en este caso particular, el itinerario de estudios en carreras universitarias. En este caso, un grupo de estudiantes está cursando una clase de español en el nivel intermedio medio y alto (ACTFL, 2012), y cuya concentración de estudios son diferentes maestrías (política internacional, medioambiente, negocios) en una universidad en Estados Unidos. Los estudiantes tuvieron la oportunidad de trabajar con contenidos afines a sus carreras durante un semestre. Como parte de la medición de las habilidades, el instructor a cargo de los cursos utilizó el portafolio de lenguas europeo adaptado al contexto universitario estadounidense.

Palabras Claves: autonomía; desarrollo curricular; portafolios; AICLE; motivación.

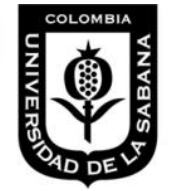

Oliva Parera, P. M., \& Nuñez Delgado, M. P. (2014). La integración de lengua y contenidos afines a diferentes carreras universitarias. Latin American Journal of Content and Language Integrated Learning, 7(2), 103-122. doi:10.5294/laclil.2014.7.2.6 elSSN 2322-9721. 


\section{INTRODUCCIÓN}

En los últimos años, el aprendizaje integrado de contenidos y lenguas (AICLE) ha demostrado su eficacia en la enseñanza de idiomas a la hora de desarrollar en los alumnos habilidades lingüísticas usando la lengua extranjera como vehículo para el aprendizaje de diferentes contenidos (Coyle, Hood \& Marsh, 2010; Hoyos Pérez, 2013) en todos los niveles educativos. Este fenómeno ha tenido un crecimiento sostenido durante los últimos veinte años en instituciones tanto en el continente europeo como en el americano (Breeze, 2014; Doiz, Lasagabaster \& Sierra, 2013; Fortanet-Gómez, 2013; Grabbe \& Stoller, 1997; Woźniak, 2013; Madrid \& Madrid, 2014).

Debido a este desarrollo del AICLE en los Estados Unidos, se ha notado una profusión de programas en diferentes universidades en el país (Jourdenais \& Springer, 2005), efecto que parece ser coincidente con un mayor interés en el aprendizaje de idiomas. La adopción de esta metodología y la apertura que tiene en el país, sobre todo en el ambiente universitario, no solo se debe a la maleabilidad que tiene en diferentes contextos de aula (Crandall \& Kaufman, 2003; Gómez Camacho, 2013), sino que también provee a los estudiantes de la L2 una competencia lingüística tendiente al desarrollo del lenguaje académico o Cognitive Academic Language Proficiency (Cummins, 1989).

En este artículo nos proponemos, por un lado, a describir el desarrollo de una unidad temática relacionada con contenidos afines a las carreras de los aprendientes que toman un curso de español como lengua extranjera en una universidad estadounidense, nivel intermedio bajo y medio según ACTFL (2012). Por otro lado, nos proponemos a reflexionar a través de esta investigación-acción, una serie de interrogantes que nos llevará a tomar decisiones con respecto a la adopción de este tipo de metodología en clases futuras. Teniendo esto en cuenta, nuestro estudio intentará responder las siguientes preguntas de investigación:

- ¿Es posible la integración de contenidos con contenido relevante a las carreras de maestrías de los aprendientes?

- ¿Puede utilizarse el enfoque de las 6 Tes para llevar a cabo dicha integración?

- ¿Qué alcances tiene en la motivación de los estudiantes la integración de lengua y contenidos? 
- ¿Es posible esta integración de lengua y contenidos con estudiantes cuyos niveles son intermedio bajo/medio?

\section{La integración de contenidos y la motivación}

Uno de los rasgos de la integración de contenidos y lengua es que no existe una sola manera para llevarla a cabo, esto dependerá de los recursos de los establecimientos educativos que lo implementan (Lasagabaster \& Ruíz de Zarobe, 2010; Shaw, 1997). También hay que notar que se mencionan ciertos inconvenientes a la hora de seleccionar el contenido, y este es uno de los retos que presenta la metodología, sobre todo si se trabaja con estudiantes adultos universitarios de posgrado. Esto se debe a que hay en realidad dos tendencias: la mayoría del profesorado está entrenado para enseñar lengua y no el contenido que a veces resulta ser muy específico. También tenemos casos de profesores expertos en contenidos como matemáticas, política, etcétera y no están preparados para la enseñanza de la lengua (Lyster \& Balinger, 2011).

Una iniciativa para superar este desafío para los profesores expertos en la enseñanza de lenguas que enseñen clases AICLE, es la preparación con respecto al contenido a enseñar por medio del autoestudio y preparación del contenido (Nuñez Delgado, González Vázquez \& Trujillo Sáez, 2006), como así también la indagación de los gustos e intereses de los estudiantes que se tendrán en cuenta a la hora la selección de material y planificación del andamiaje gramatical como se explicará más adelante.

En cuanto a la motivación, un aspecto para tener en cuenta es que el AICLE, en este sentido, parece ser el contexto ideal para fomentar la motivación por despertar el interés de los aprendientes a la hora de expresarse de una manera más natural en una clase de lengua (Dupuy, 2000). Creemos conveniente hacer mención que hacia los años 80, comienza un debate entre los estudiosos de la motivación (McDonough, 1981; Clément \& Kruidenier, 1983) con respecto al aspecto "integrativo" de la motivación. Este aspecto integrativo cobra importancia en los estudios actuales de la motivación al considerar al aprendiente y su propia perspectiva en la comunidad global en lugar de ver la comunidad global como un agente externo Ushioda (2006). Este nuevo aspecto de la motivación, que tiene en cuenta diferentes perspectivas o identidades que los alumnos traen a la clase, converge en un nuevo modelo motivacional propuesto 
por Dörnyei y denominado sistema motivacional del yo o "motivational self system" (Dörnyei, 2005; 2009). El autor diferencia tres componentes, a saber:

a) El yo ideal o "Ideal L2 self": que se relaciona con la meta ideal de habla que, en este caso, el alumno de L2 desea tener. Este aspecto estás más relacionado con lo intrínseco.

b) El yo del "deber" u "Ought-to L2 self": es el componente que está relacionado con lo que uno "debe hacer" para prevenir resultados negativos. Esta es la dimensión que está relacionada más con la motivación extrínseca.

c) La experiencia de la L2 o "L2 Learning experience": en este caso la experiencia de aprendizaje es la encargada de sustentar la imagen del yo ideal iniciada por el aprendiente pero para que ésta sea mantenida debe haber una buena experiencia de aprendizaje.

Una orientación similar ha sido rescatada también por Benson (2007) cuando expresa que la motivación es fundamental en el aprendizaje de una lengua y es inherente al trabajo autónomo del aprendiz mientras que la identidad está más relacionada con la meta final de esa labor autónoma del alumno. Si se tiene en cuenta este punto de vista, llevado a la realidad del aula, se puede percibir una situación ideal en la enseñanza de lengua y contenidos que contemple lo que los alumnos tienen que decir además de sus intereses y sus necesidades en un contexto que sea desafiante. A continuación nos referiremos a otro componente necesario en el AICLE que es el instructor.

\section{El rol ideal del docente en las clases AICLE y el andamiaje}

El rol del instructor en las clases AICLE será principalmente de conducir al estudiante a desarrollar su propia autonomía actuando como el nexo entre lo que el estudiante puede hacer por sí mismo y lo que puede lograr en cuanto a los objetivos en potencia con respecto a la adquisición de las habilidades de la lengua (Vygotski, 1978). Para esto contará con un arsenal de materiales en su repertorio con el fin de llevar a cabo este cometido. En primer lugar, recurrirá al andamiaje o "scaffolding" (Bruner, 1983; van Lier, 2004) a partir del que brindará al estudiante el apoyo necesario para que la experiencia de aprendizaje sea placentera y contribuya al afianzamiento de las destrezas y haciendo el enfoque necesario en la forma o en las formas durante el andamiaje gramatical. Esa comunión mutua entre alumno y profesor durante el andamiaje es esencial para 
el desarrollo de la autonomía aparte de ser constitutiva de la teoría de aprendizaje sociocultural muy debatida actualmente por el diálogo entre los que participan en el proceso de enseñanza-aprendizaje (Lantolf \& Thorne, 2006). Se pasará ahora a la descripción de una herramienta que se ha implementado en la clase AICLE, y ha resultado ser una aliada a la hora de evaluar diferentes logros del estudiante.

\section{El portafolio de lenguas}

Si bien se ha utilizado el portafolio en diferentes carreras, en el aprendizaje de lenguas será definido según Artel \& Spandel (1992) citado en Klenowsky (2012) como:

La colección de los trabajos escritos de un estudiante en la que se muestran claramente el esfuerzo, el progreso y los logros que se han claramente obtenido en ciertas áreas específicas. Esta colección debe incluir la intervención del estudiante en la selección del contenido de los textos, los criterios para su selección, los criterios para juzgar los logros y la evidencia de auto-reflexión por parte del estudiante. (p. 13)

En esta definición es importante resaltar el aspecto evaluativo del portafolio y también el hecho de que el aprendiente es parte dinámica de esta tarea ya que necesita coleccionar los trabajos que ha de presentar, reflexionar sobre los mismos y autoevaluarse. Si bien el portafolios europeo fue desarrollado en conjunto con el "El Marco Común Europeo de Referencia para las Lenguas" (MCER) (Council of Europe, 2001) y según el MCER, uno de los objetivos es facilitar los estudios de la lengua a estudiantes en la comunidad europea, el siguiente portafolios fue adaptado teniendo en cuenta la edad adulta de los aprendientes en un contexto universitario de posgrado en una universidad estadounidense. Este portafolio constó de:

1) Un análisis de necesidades: el estudiante explicó brevemente su historia con la lengua meta como así también los objetivos a corto y a largo plazo de la clase. En el portafolio europeo esta sección es denominada "pasaporte". El nombre, en este caso, ha sido cambiado para contextualizarlo al ambiente universitario de posgrado.

2) La segunda sección se denomina "la biografía lingüística”. En esta segunda sección del portafolio los estudiantes reflexionaron y se autoevaluaron por medio de diferentes trabajos. Los estudiantes al finalizar el portafolios y a 
modo de cierre, escribieron un ensayo de autoevaluación sobre su progreso durante el semestre.

3) En la tercera y última sección se encuentra el "dossier" donde los estudiantes colocaron a modo de archivo los trabajos que querían utilizar para mostrar sus habilidades y/o conocimientos lingüísticos.

La implementación de este portafolio europeo en el curso de español fue explicado en el anexo (1).

Teniendo en cuenta lo planteado en la introducción. Es decir, en primer lugar, recabar información para la toma de decisiones con respecto a clases futuras a través de la metodología AICLE en la institución y, en segundo termino, intentar responder a las preguntas que motivaron el siguiente estudio; se decidió llevar a cabo una investigación-acción y de ahí en más adoptar las medidas necesarias para hacer los cambios correspondientes. A partir de esto, pasaremos ahora la explicación de la metodología e instrumentos utilizados.

\section{METODOLOGÍA}

Debido a la naturaleza de lo que se intentó explorar, utilizamos el diseño de grupo único con post-test (Carrasco \& Calderero Hernández, 2000). Se recurrió también al uso de metodología cualitativa. En este sentido se entiende que los resultados de esta experiencia podrían ser extrapolados a otros contextos de aprendizaje si se tuviera un diseño cuantitativo. No obstante, no se cree conveniente desdeñar los resultados de una experiencia de este tipo sobre todo cuando clarifican por medio de ellos situaciones de aula (Nunan \& Bailey, 2009).

\section{Participantes}

Los participantes fueron 13 estudiantes que cursaban diferentes maestrías (política internacional, medioambiente, negocios) en Monterey Institute of International Studies en California. Todos eran estadounidenses excepto uno que era italiano. La edad promedio de los estudiantes fue 31 años. El $62 \%$ de la muestra eran mujeres. La clase se denominó "Cultura y lengua hispana" y fue la primera clase que se dictó en la institución universitaria en el nivel (intermedio bajo/medio) en la institución. La clase tuvo un encuentro de dos horas de reloj dos veces por semana. 


\section{Instrumentos utilizados y procedimiento}

Se utilizó un portafolio para la recolección de los datos. La implementación del mismo en el curso fue explicada, como se ha dicho anteriormente, en el Anexo 1. De este portafolio, se recurrió al análisis cualitativo de la información que aparecía en el ensayo de auto-evaluación de la bibliografía lingüística. Para triangular la siguiente investigación, se utilizó la información brindada por los estudiantes pertenecientes a la evaluación oficial de la institución donde se llevó a cabo el estudio. Esta evaluación oficial es completamente anónima y los estudiantes la completaron en línea durante la última semana de clases. La misma fue entregada al profesor un mes después de la finalización del semestre.

\section{Materiales utilizados en el tratamiento}

En cuanto al material utilizado en el tratamiento, es decir en la clase AICLE, recurrimos a material auténtico obtenido de buscadores en la universidad donde se llevó a cabo el estudio y libros de textos especializados. También se recurrió a material escrito por el docente que está en proceso de publicación. En cuanto a la temática, dentro del concepto los derechos humanos, los temas estudiados fueron los siguientes: "las desapariciones en Argentina durante los setenta" y "violencia de género". Cada uno de estos temas se planificó para el transcurso de dos semanas pero a veces se extendió a una tercera semana dependiendo en los intereses de los alumnos. Se puede leer una descripción de parte del material que se refiere a la temática de los derechos humanos en el Anexo 2.

\section{RESULTADOS}

Teniendo en cuenta la primer pregunta que motivó la presente investigación, podemos afirmar que, en primer lugar, se identificó el área del problema y esto significó saber si fue posible la integración de contenidos relacionados a los itinerarios de las carreras de los aprendientes a la clase de español denominada "Cultura y Lengua Hispana". En otras palabras, estas carreras de posgrados tienen un enfoque en medioambiente, política internacional y negocios. A partir de esto, se creyó conveniente explorar la posibilidad de dicha integración con estas áreas de estudio. Para empezar, decidimos indagar en contenidos relevantes a estos itinerarios que podríamos incluir en la clase. Por este motivo, organizamos reuniones con colegas que son expertos en clases de contenidos relacionados con 
política internacional, medio ambiente y negocios. Posteriormente, decidimos que la temática general de la clase se enfocaría en tres conceptos a lo largo del semestre. Estos conceptos fueron: los aborígenes, el medio ambiente y los derechos humanos.

En segundo término, creímos importante conocer si esta integración se podía llevar a cabo a través del enfoque de las 6 Tes o "Six T's approach" (Grabbe \& Stoller, 1997). En otras palabras, una vez seleccionados los grandes temas o conceptos, se identificaron los textos a utilizar, se buscaron diferentes temas o topics que se desprendieran de esos textos. Se identificaron hilos conductores o threads en común con las carreras mencionadas anteriormente. También se planificó el andamiaje gramatical o tasks basado en el material encontrado y se planificaron posibles transiciones o transitions desde el punto de vista temático. Este trabajo implicó una búsqueda en la biblioteca de la institución de revistas especializadas y sitios web accesibles para la identificación de material auténtico a utilizar.

En tercer lugar, consideramos necesario observar los alcances de esta integración en la motivación. Para esto, se analizaron todas las oraciones que describían el yo ideal o ideal L2-self del ensayo auto-evaluativo final perteneciente a la bibliografía lingüística del portafolios de los 13 participantes. Una vez analizadas las oraciones, se pudo observar que las respuestas dadas por ellos mismos estaban íntimamente ligadas a razones relacionadas a su carrera en particular, es decir, a sus campos de trabajo y estudio. En otras palabras, el $85 \%$ de los estudiantes (todos excepto 2) quieren aprender y mejorar la L2 para un mejor desempeño en los empleos que tengan a futuro. Las oraciones analizadas extraídas y analizadas pueden leerse en el Anexo 3.

En cuarto término, nuestra intención era también saber si esta integración se podía realizar con estudiantes de nivel (intermedio bajo /medio). Este es el primer curso de lenguas que los estudiantes tomaron una vez que fueron aceptados a la universidad para hacer su correspondiente maestría. Es posible afirmar que la integración se pudo llevar a cabo y fue exitosa teniendo en cuenta dos elementos: la perspectiva del profesor y la del alumno. En cuanto a la primera, todos los alumnos en el grupo que finalizaron el curso de español y fueron promovidos al curso siguiente con una nota promedio satisfactoria. Sin embargo, la tarea ha sido desafiante. Para ilustrar este punto y a modo de ejemplo, cuando empezamos a trabajar el concepto de derechos humanos cuya actividad se encuentra, como 
dijimos anteriormente, en el anexo (2), los estudiantes se encontraron motivados desde el principio por medio de la participación activa durante la actividad del torbellino de ideas. Se notó que los estudiantes poseían el conocimiento y utilizaban la lengua como vehículo para expresar sus pensamientos sin prestar mucha atención a la gramática de las frases u oraciones expresadas. En esta línea se ve que el aporte de esta temática de los derechos humanos cuadra con lo expresado por Dörnyei (2005) en el sentido de que a menos aptitud por parte del alumno, mayor motivación se necesitará en el aprendizaje. En otras palabras, la temática ha resultado lo suficientemente motivadora para que los estudiantes se expresen y el docente, sobre todo en este nivel, les haga prestar atención a la forma (Gibbons, 2002).

Mencionamos anteriormente que la integración resultó exitosa desde el punto de vista del profesor por lo hasta aquí mencionado. Con respecto al punto de vista del alumno, los estudiantes en general se mostraron satisfechos con los resultados de acuerdo a lo expresado en la evaluación final del profesor (triangulación). Cabe mencionar que dicha evaluación ha sido contestada por 10 alumnos de los 13 que tenía el curso. En el próximo apartado, discutiremos algunos de los retos a los que nos enfrentamos trabajando con la metodología en este nivel de lengua.

\section{DISCUSIÓN}

Creemos necesario remarcar que nuestra tarea ha sido desafiante en tres puntos principales. En el primero, tenemos que mencionar las horas de preparación del andamiaje gramatical que acompañaban al material auténtico utilizado en la enseñanza (cortos, recortes de periódicos, textos para escuchar, etcétera.) como así también la preparación de exámenes donde se integró la gramática y el contenido visto en los temas. A partir de esta experiencia, se empezó a organizar un banco de datos con diferentes contenidos y gramática para su uso futuro. En segundo lugar, tuvimos que realizar ajustes a la planificación por el tiempo que llevaban las actividades. Había temas donde los estudiantes se notaban muy motivados en el diálogo y tomaban más tiempo en las presentaciones que realizaban por las preguntas que luego hacían. Percibimos esto como una experiencia positiva y muy característica de estudiantes adultos en un nivel de posgrado. En tercer término, teniendo en cuenta el tipo de estudiantes, una de las actividades utilizadas para propiciar no solo su motivación sino también 
autonomía en este tipo de metodología, consistió en la investigación propia de temas relacionados con los temas de las clases. Es decir, al estudiarse el medio ambiente, los alumnos tenían la libertad de elegir algún tema para presentar a la clase por 10 minutos. Esto requirió una preparación previa del alumno por medio de la investigación y preparación de un PowerPoint para toda la clase. Otros de los aspectos importantes es que por medio de esta integración, ha sido posible motivar a los aprendientes porque en las clases respectivas, se ha observado cómo a partir del contenido, los alumnos eran desafiados en su progreso hacia una mayor aptitud lingüística por el hecho de que se inclinaban a participar y expresar diferentes perspectivas desde su punto de vista personal. En la clase se observó en varias instancias la manera en la que los estudiantes discutían y negociaban significados de una manera cooperativa a partir de experiencias previas de trabajo que los hacía aprender de los demás y a pensar a través de la lengua (Gibbons, 2002).

A la luz de los resultados y para concluir, queremos expresar que se ha podido observar también como se ha logrado la integración a partir de este nivel de lengua y ver progreso en el desarrollo del lenguaje académico (Cummins, 1989). Para esto, fue necesario, concientizar a los alumnos de las diferentes formas a través del andamiaje gramatical. Esto se hace más necesario aún, en clases que siguen este tipo de formatos ya que dependiendo de los textos utilizados, el contenido es el que impone la gramática. A través de la incorporación del portafolios de lenguas europeo adaptado a un contexto estadounidense y universitario, el alumnado tuvo la oportunidad de "expresarse como ellos quieren" o "speak as themselves" (Ushioda, 2005), en la sección correspondiente a la bibliografía lingüística del portafolios. El análisis de las oraciones relacionadas al yo ideal o "Ideal L2 self" (Dörnyei, 2009; Dörnyei \& Ushioda, 2011) ha permitido observar que la mayoría de los estudiantes vinculan la futura imagen de la L2 con trabajos o disciplinas a desarrollar una vez que se gradúen. En este sentido, se observa cómo en este tipo de clases se fomenta la motivación a través de contenidos afines a su itinerario de carrera. A modo de limitaciones en el presente trabajo investigativo se desea hacer notar el aspecto cualitativo que no permite la extrapolación de los resultados a otros estudios de esta índole por lo que convendría en el futuro un diseño de estudio que contemple un aspecto cuantitativo de la muestra como así también un grupo de control. 


\section{REFERENCES}

ACTFL. (2012). ACTFL proficiency guidelines. Retreived from http://www.actfl.org/publications/guidelines-and-manuals/actflproficiency-guidelines-2012

Arter, J. \& Spandel, V. (1992). Using portfolios of student work in instruction and assessment. Portland, OR: Northwest Regional Education Laboratory.

Benson, P. (2007). Autonomy in language teaching and learning. Language Teaching, 40, 21-40.

Breeze, R. (2014). Identifying student needs in English-medium university courses. In R. Breeze, C. Llamas Saiz, C. Martinez Pasamar, \& C. Tabernero Sala (Eds.), Integration of theory and practice in CLIL (pp. 143-160). Amsterdam, the Netherlands: Rodopi.

Bruner, J. (1983). Child's talk: Learning to use language. Oxford, UK: Oxford University Press.

Carrasco, J. \& Calderero Hernández, J. (2000). Aprendo a investigar en educación. Madrid, Spain: RIALP.

Clément, R. \& Kruidenier, B. (1983). Orientations in second language acquisition: The effects of ethnicity, milieu, and target language on their emergence. Language Learning, 33, 273-291.

Council of Europe. (2001). Common European framework of reference for languages: Learning, teaching, assessment. Cambridge, UK: Cambridge University Press.

Coyle, D., Hood, P., \& Marsh, D. (2010). CLIL: Content and language integrated learning. Cambridge, England: Cambridge University Press.

Crandall, J. \& Kaufman, D. (2003). Content-based instruction in higher education settings. Alexandria, VA: TESOL.

Cummins, J. (1989). Empowering minority students. Sacramento, CA: California Association for Bilingual Education.

Doughty, C., \& Varela, E. (1998). Communicative focus on form. In C. Doughty \& J. Williams (Eds.), Focus on form in classroom second language acquisition (pp. 114-138). Cambridge, UK: Cambridge University Press.

Dörnyei, Z. \& Ushioda, E. (2011). Teaching and researching motivation. Harlow, UK: Longman. 
Dörnyei, Z. (2009). The L2 motivational self system. In Z. Dörnyei \& E. Ushioda (Eds.), Motivation, language identity and the L2 self (pp. 9-42). Bristol, UK: Multilingual Matters.

Dörnyei, Z. (2005). The psychology of the language learner: Individual differences in second language acquisition. Mahwah, NJ: Lawrence Erlbaum.

Doiz, A., Lasagabaster, D., \& Sierra, J. M., (Eds). (2013) English-medium instruction at universities: Global challenges. Bristol,UK: Multilingual Matters.

Dupuy, B. (2000). Content-based instruction: Can it help ease the transition from beginning to advanced foreign language classes? Foreign Language Annal, 33, 205-223.

Fortanet-Gómez, I. (2013). CLIL in higher education: Towards a multilingual language policy. New York, NY: Multilingual Matters.

Gibbons, P. (2002). Scaffolding language, scaffolding learning: Teaching ESL children in the mainstream classroom. Porstmouth, NH: Heinemann.

Gómez Camacho, A. (2013). El aprendizaje integrado de la lengua española y los contenidos de áreas no lingüísticas en los proyectos lingüísticos de centro. Porta Linguarum, 20, 103-115.

Grabbe, W., \& Stoller, F. (1997). A six T's approach to content based instruction. In M. Snow y D. Brinton. (Eds.), The content-based classroom: Perspectives on integrating language and content (pp. 78-94). New York: Longman.

Hoyos Pérez, M. (2013). La enseñanza integrada de lenguas y contenidos en formación profesional. Revista de Lenguas para Fines Específicos, 19, 109145.

Jourdenais, R., \& Springer, S. (Eds). (2005). Content tasks and projects in the language classroom: 2004 conference proceedings. Monterey, CA: Monterey Institute of International Studies.

Klenowsky, V. (2012). Desarrollo de portafolios: Para el aprendizaje y la evaluación. Madrid, Spain: Narcea, SA.

Lantolf, J., \& Thorne, S. (2006). Sociocultural theory and second language acquisition. In W. Van Patten \& J. Williams (Eds.), Theories in second language acquisition (pp. 201-224). Mahwah, NJ: Lawrence Erlbaum Associates. 
Lasagabaster, D., \& Zarobe, Y. R. de (Eds.). (2010). CLIL in Spain: Implementation, results and teacher training. Newcastle upon Tyne, UK: Cambridge Scholars Publishing.

Lyster, R. \& Ballinger S. (2011). Content-based language teaching: Convergent concerns across divergent contexts. Language Teaching Research, 15, 279288.

Madrid, Manrique, M., \& Madrid, Fernández, D. (2014). La formación inicial del profesorado para la educación bilingüe. Granada, Spain: la Universidad de Granada.

McDonough, S. (1981). Psychology in foreign language teaching. London, UK: Allen and Unwin.

Nunan, D. \& Bailey, K. (2009). Exploring second language classroom research: A comprehensive guide. Boston, MA: Heinle/Cengage Learning.

Nuñez Delgado, M. P., González Vázquez A., \& Trujillo Sáez F. (2006). La formación del profesorado de español como Segunda Lengua: Situación actual y propuestas. Textos de Didáctica de la Lengua y la Literatura, 42, 6580 .

Papai, N. (2000). Literacy development through content-based instruction: A case study. Working Papers in Educational Linguistics, 16, 81-95.

Shaw, P, (1997). With one stone: Models of instruction and their curricular implications in an advanced content-based foreign language program. In S. B. Stryker \& B. L. Leaver (Eds.), Content-Based instruction in foreign language education: Models and methods (pp. 261-282). Washington, DC: Georgetown University Press.

Ushioda, E. (2006). Language motivation in a reconfigured Europe: Access, identity, autonomy. Fournal of Multilingual \& Multicultural Development, 27, $148-161$.

Van Lier, L. (2004). The ecology and semiotics of language learning: A sociocultural perspective. Boston, MA: Kluwer Academic.

Vygotsky, L. S. (1978). Mind in society: The development of higher psychological processes. (M. Cole, V. John-Steiner, S. Scribner, \& E. Souberman, Eds.). Cambridge, MA: Harvard University Press.

Woźniak, M. (2013). CLIL in pharmacy: A case of collaboration between content and language lecturers. Language Value, 5, 107-128. 


\section{ANEXO 1}

\section{Presentación del portafolio a la clase}

La presentación del portafolio se realizó el primer día de clases. Se les explicó las diferentes partes del portafolio como así también se les aclaró que tendría un valor de $25 \%$ en la nota final. Luego, el instructor habló brevemente de las destrezas que existen en el aprendizaje de una lengua extranjera y de la importancia del desarrollo de la competencia comunicativa en la L2. También se tomó una de las destrezas y dio un ejemplo de cómo se puede demostrar progreso en una competencia utilizando el portafolio.

\section{Repasando algunas estrategias y el portafolios}

Tres semanas después de haber presentado el portafolio, el instructor decidió abordar el tema de las estrategias de aprendizaje para estudiar el vocabulario. Los alumnos realizaron la siguiente actividad:

\section{Actividad}

Completa las preguntas y luego trabaja en pares para discutir las respuestas.

1. ¿Usas el diccionario?

2. ¿Traduces todo al inglés?

3. ¿Repites en voz alta?

4. ¿Dibujas las palabras para recordarlas?

5. ¿Dibujas diagramas y gráficos?

6. ¿Haces listas de palabras en español e inglés para memorizar las palabras?

7. Cuando escuchas la definición de una palabra, ¿la escribes inmediatamente con ejemplos?

8. ¿Prefieres ensayar lo que vas a decir en español antes de decirlo?

9. ¿Lees artículos en español para ampliar el vocabulario? 


\section{ANEXO 2}

\section{Temática de los derechos humanos}

Para empezar a tratar este tema, se realizó una discusión sobre ello. Para incentivar la discusión, el instructor escribió en la pizarra lo siguiente:

\section{Los derechos humanos (DDHH)}

De esta manera comenzó un torbellino de ideas a través de preguntas que él hacía para obtener algunas respuestas que escribía en la pizarra a medida que los estudiantes levantaban la mano y participaban. Algunas preguntas que se utilizaron fueron las siguientes:

- ¿Qué son los derechos humanos? ¿Son importantes y por qué?

- ¿Piensas que tus derechos humanos aquí en los EEUU son respetados o no? ¿Por qué?

- ¿Qué piensas tú cuando los derechos humanos no son respetados?

A medida que los estudiantes respondían, el instructor escribía en la pizarra algunas palabras que eran prácticamente nuevas para el alumnado:

- $\quad$ Tienes los derechos al nacer y en algunos países antes de nacer.

- $\quad$ Son inherentes a la persona.

\section{Los derechos humanos}

Vivir en democracia supone un respeto a los derechos humanos. Algunas veces existe un atropello de los derechos por el gobierno.

Se observó que algunos alumnos tomaban notas de lo que el profesor escribía en la pizarra y hubo un estudiante que preguntó por la construcción "al nacer”. El profesor le respondió lo que significaba en la L1 del estudiante. Otro estudiante preguntó por la palabra "atropello" y el profesor contestó "abuso".

Luego el profesor se dispuso a trabajar en la siguiente actividad para la cual les pidió a los alumnos juntarse en pares.

\section{Trabajo en pares: Actividad}

Lee las siguientes palabras y decide cuáles pertenecen a un país en "democracia" o "no en democracia". Explica el por qué. 
- ¡Marcela! Necesitas bajar la música. Las canciones de Mercedes Sosa están prohibidas. Ella está proscripta en el país.

- La identidad de una persona es algo inherente a la persona humana. Los gobiernos de los países necesitan protegerla.

- ¿Qué político te gusta más? ¿Te gustan las buenas ideas de los partidos políticos este año?

- $\quad$ El golpe de estado en Chile tuvo muchas víctimas, muchos presos políticos.

- Las organizaciones no gubernamentales(ONG) que defienden a los aborígenes decidieron estar cerca de la presidenta.

- $\quad$ Este fin de semana tenemos veda electoral porque el domingo se votará a los nuevos candidatos.

- La dictadura en este país es cosa del pasado. Lo importante ahora es la memoria.

- La junta militar va a decidir las nuevas leyes en el país.

Los estudiantes organizan las oraciones bajo estas dos categorías:

\begin{tabular}{|l|l|}
\hline País que vive en democracia & País que no vive en democracia \\
\hline
\end{tabular}

El instructor les pide a los estudiantes agrupados en pares juntarse con otros pares para comparar las respuestas. A continuación el instructor les pidió a los estudiantes que leyeran la siguiente carta y se preparasen para responder a preguntas.

Estimados tíos,

Estoy tan contento de saber que los he encontrado finalmente. Mi búsqueda parecía no tener fin. Estoy casado con una española desde hace 5 años. Ester y yo tenemos 3 hijos. Manuel, Nicolás y Carlota. Manuel tiene 6, Nicolás 4 y la pichoncita tiene 2. Tengo muchos recuerdos de mi infancia en Argentina. Me acuerdo de que mis padres y yo nos mudábamos mucho por aquel entonces. El año pasado tuve mi primer contacto con este grupo de personas que vinieron a España que me contactaron a través de Facebook. Decidí juntarme con ellos una tarde y conté mucho de estos recuerdos a estas comisiones de la verdad. Siempre voy a recordar mi último día en Argentina. Un día, al salir de la escuela, vi a mi madre esperándome. Me sorprendió un poco pues siempre el transporte escolar me llevaba de la escuela a mi casa todos los días porque mi madre trabajaba en ese horario. Inmediatamente, me di cuenta de que algo pasaba por la mirada en el rostro de mi madre. No pregunté nada... mi madre me abrazó y me dijo " Carlitos... vamos a irnos a un lugar muy lejos”. Yo le pregunté: “¿y papá, dónde está? Ella me contestó sin mirarme a los ojos y me dijo: "vinieron y se lo llevaron”. Sentí su pena en el corazón y no le quise preguntar más hasta que 
tuve la edad de 13 años. De la escuela nos llevó un amigo de la familia de mi padre hasta el aeropuerto de Ezeiza a toda velocidad. Al llegar al aeropuerto y luego de una despedida más que fugaz, caminamos hacia el avión. Recuerdo que luego de viajar toda la noche aterrizamos en Madrid donde nos esperaba una familia de argentinos refugiados que mi madre no conocía. Pasaron los años y al cumplir los 23 años falleció mi madre. No me interesó volver más a la Argentina. En España me hice de amigos rápidamente en la escuela y siempre la sentí como mi hogar. Pero los años pasaban y dicen que la sangre tira... siempre me preguntaba lo que mi madre y yo habíamos dejado atrás. Siempre pensaba cuán diferente mi vida hubiese sido si nos habríamos quedado allá... Mirando todas estas noticias sobre la democracia en Argentina y el castigo a los represores de la junta... Empecé a recordar muchas cosas y a peguntarme sobre mis familiares argentinos. Una de las cosas que recordé eran los asados tan ricos en la casa de ustedes. Esos recuerdos se me habían borrado completamente hasta ahora. El año pasado, después de ver un comercial en la televisión sobre los desaparecidos y esta asociación en Europa; me dirigí a la ONG “abuelas de plaza y de mayo" y decidí contar mi historia. Me llamaron nuevamente dos semanas después y me hablaron de mis queridos tíos que todavía querían saber sobre mi paradero. Es así como los encontré o mejor dicho me encontraron. Ester y yo tenemos planeado visitarlos para Navidad. Les mando un beso grande a los dos y nos veremos dentro de poco. Besos y abrazos de su sobrino perdido, Carlitos Saldaña.

Contesta las preguntas basadas en la carta:

1) ¿Cómo es que toman contacto Carlos y su familia en Argentina?

2) ¿Qué piensas que le pasó al padre de Carlos?

3) ¿Por qué piensas que su madre y Carlos decidieron salir de la Argentina?

4) ¿Tiene buenos recuerdos Carlos de su infancia? Explica.

5) ¿Por qué piensas tú que Carlos no le pregunta a su madre sobre su padre?

6) ¿Qué recuerdos tiene Carlos de sus tíos argentinos?

Mira las siguientes frases $\mathrm{u}$ oraciones y marca la mejor alternativa según el contexto

1) Mi búsqueda parecía no tener fin.

2) El narrador buscó a su familia por un largo tiempo.

3) El narrador decidió abandonar la idea de buscar a su familia.

4) El narrador le puso fin a la idea de buscar a su familia.

5) El narrador buscó y encontró a su familia.

6) Me acuerdo de que mis padres y yo nos mudábamos mucho por aquel entonces. 
7) Mis padres y yo nos comprábamos mucha ropa.

8) Nos acordábamos de comprar mucho.

9) Cambiábamos de domicilio con frecuencia.

10) Mis padres y yo nos movíamos adentro de la casa.

11) Decidí juntarme con ellos una tarde y conté mucho de estos recuerdos a estas comisiones de la verdad.

12) Les hablé a las Comisiones de la verdad acerca de mi familia española.

13) Me reuní con las personas que eran parte de las Comisiones de la verdad para pasar el rato.

14) Me reuní con la organización para contar mi historia de vida.

15) Me reuní con las personas para contarles sobre mi vida en Argentina y salí del país por cuestiones políticas.

16) Al llegar al aeropuerto y luego de una despedida más que fugaz, caminamos hacia el avión.

17) Cuando llegamos al aeropuerto, nos fuimos rápido sin despedirnos.

18) Al llegar a destino, nos besamos y abrazamos rápidamente y caminamos hacia el avión.

19) Al llegar al aeropuerto, nos fuimos al avión caminando.

20) Cuando llegamos al aeropuerto, mi madre se retiró sin decir adiós.

21) Pero los años pasaban y dicen que la sangre tira. Siempre me preguntaba lo que mi madre y yo habíamos dejado atrás.

22) La familia es importante.

23) La familia del autor fue asesinada por los militares.

24) Hubo mucha sangre en la Argentina por años.

25) El autor se acordaba de su vida en familia en Argentina.

26) Siempre pensaba cuán diferente mi vida hubiese sido si nos hubiéramos quedado allá.

27) El autor pensaba sobre su vida en Argentina.

28) El autor se imagina cómo es la vida es Argentina.

29) El autor piensa sobre sus familiares en Argentina.

30) El autor piensa como sería la vida en Argentina si no estuviera en España.

Los estudiantes trabajaron y luego las respuestas se verificaron oralmente en la clase. Para finalizar la unidad el instructor a cargo del grupo les pidió a los estudiantes que buscaran información para llevar a cabo el siguiente debate. Se 
les dijo que ellos podían estar en un grupo o en otro y que necesitarían información para justificar una como otra posición.

\section{$\underline{\text { Debate }}$}

"En Latinoamérica los diferentes gobiernos respetan los derechos humanos."

En esta ocasión el docente a cargo del grupo dividió al grupo en dos partes. Los estudiantes tendrían 10 minutos para poner sus ideas en común. Los grupos trabajaron en forma independiente. Mientras los grupos realizaban la actividad, los estudiantes hacían preguntas al instructor con respecto a vocabulario. Una vez que los estudiantes pusieron sus ideas en común, el profesor dio comienzo al debate. Es importante notar que el instructor trabajó anteriormente con los alumnos diferentes maneras para expresar, concordar y refutar puntos de vista.

\section{ANEXO 3}

\section{Oraciones extraídas de la ensayo (bibliografía lingüística)}

"Seguiré practicando el español viajando a países de habla hispana y buscaré oportunidades para usarlo en los Estados Unidos también. Quisiera poder leer Cien años de soledad en español. Me gustaría aprender a entender, escribir y hablar con más fluidez”. (1)

"Quiero usar el español en una manera profesional. Planeo trabajar con latinoamericanos y quiero estar preparada a tener una conversación con cualquier persona." ( 2)

"Mis objetivos a largo plazo son que podré comunicar con otros en el idioma con nivel suficiente para trabajar en una organización.” (3)

"En el futuro, espero asistir clases que pertenezca a los negocios. Ya que soy estudiante de negocios, quiero aprender cosas de la profesión.” (4)

"Mi escritura, mi escucha y aun mi hablar han mejorado enormemente. Trataré de aprender más vocabulario y unos términos español sobre los temas de la economía para poder usar esta lengua en mi futura carrera.” (5)

"Un objetivo a largo plazo por esta clase es expresarme con facilidad en una manera profesional. Me gustaría hablar sin miedo y hesitación con familia, amigos y en un trabajo que tenga en el futuro." (6)

"Quiero vivir en un país de habla española y trabajar con la gente en temas de medio ambiente." (7) 
"Después de graduarme, espero trabajar con las comunidades indígenas para mejorar sus situaciones económicas por desarrollo de los negocios pequeños"... "Quiero trabajar en solidaridad, colaboración y comprensión con la gente indígena. Por eso tengo que mejorar mi habilidad para escuchar y comprender tan pronto como posible." (8)

"Una experiencia muy importante para mí en el aprendizaje de la lengua fueron mis viajes con mi iglesia en Guatemala." “.. este experiencia abrió mis ojos a la oportunidad sucesiva de conectar personas con las lenguas compartidas. Voy a traer este momento a mis experiencias futuras cuando tengo conversaciones en mi trabajo de desarrollo de negocios." (9)

"Me siento más confidente con reglas de gramáticas básicas y el subjuntivo aunque todavía sean difíciles. Como una estudiante de negocio, ahora tengo más vocabulario relacionado con la economía, la sostenibilidad, y cuestiones relativas a la energía. Ahora estoy agradecido por poder comunicar mi opinión e ideas sobre estos importantes temas en español." (10)

"A largo plazo, me gustaría tener el nivel de español para poder hacer comercio con América Latina." (11)

"Hoy en día me gusta hablar en español pero hago muchas errores de gramática, especialmente cuando explico a las ideas complicadas, y casi todo el tiempo mis ideas están complicadas. Quiero hablar y escribir como un nativo." (12)

"Quiero saber más y practicar la lengua para hablar más fluido. Es importante porque voy a ir a Perú otra vez y voy a ser el responsable de varios proyectos con ONG's." (13)

\section{BIODATA}

Pablo Marcelo Oliva Parera is Assistant Professor at the Monterey Institute of International Studies in Monterey, CA (USA), a graduate school of Middlebury College, where he is currently teaching Spanish through content-based instruction. He is also a Ph.D. candidate at the University of Granada (Granada, Spain), and obtained his Master of Arts in Educational Leadership at East Carolina University in Greenville, NC (USA). E-mail: poliva@miis.edu.

Maria Pilar Nuñez Delgado is a researcher and Professor at the University of Granada (Granada, Spain), from which she also received her Ph.D. in philology. Since October 2008, she has led the research group Étimo, which researches and publishes on the didactics of the Spanish language. E-mail: ndelgado@ugr.es. 